Relations industrielles

Industrial Relations

\title{
Le boycottage
}

\section{Marie-Louis Beaulieu}

Volume 13, numéro 2, avril 1958

URI : https://id.erudit.org/iderudit/1022443ar

DOI : https://doi.org/10.7202/1022443ar

Aller au sommaire du numéro

\section{Éditeur(s)}

Département des relations industrielles de l’Université Laval

\section{ISSN}

0034-379X (imprimé)

1703-8138 (numérique)

Découvrir la revue

\section{Citer ce document}

Beaulieu, M.-L. (1958). Le boycottage. Relations industrielles / Industrial Relations, 13(2), 169-173. https://doi.org/10.7202/1022443ar
Résumé de l'article

Voici des observations judicieuses, formulées par un juriste réputé, en marge d'une décision rendue par le juge l.-J. Deslauriers à l'encontre du boycottage secondaire. Ce jugement serait le premier du genre rendu par un tribunal québécois et portant directement sur le boycottage dans les conflits de travail.
Tous droits réservés @ Département des relations industrielles de l’Université Laval, 1958
Ce document est protégé par la loi sur le droit d'auteur. L’utilisation des services d'Érudit (y compris la reproduction) est assujettie à sa politique d'utilisation que vous pouvez consulter en ligne.

https://apropos.erudit.org/fr/usagers/politique-dutilisation/ 


\title{
JURISPRUDENCE DU TRAVAIL
}

LE BOYCOTTAGE

\section{Me Marie-Louis Beaulieu}

\begin{abstract}
Voici des observations judicieuses, formulées par un juriste réputé, en marge d'une décision ${ }^{1}$ rendue par le juge I.-J. Deslauriers à l'encontre du boycottage secondaire. Ce jugement serait le premier du genre rendu par un tribunal québecois et portant directement sur le boycottage dans les conflits de travail.
\end{abstract}

\section{LES FAITS}

L'affaire est venue devant le tribunal sur une demande d'injonction de la part de Verdun Printing and Publishing Inc. contre l'Union internationale des clicheurs et électrotypeurs de Montréal, local 33 , et quelques-uns de ses officiers. Depuis plusieurs mois, les rapports entre les parties étaient l'objet de démêlés et de conflits. Des démarches avaient été faites par le syndicat pour forcer la requérante à négooier une convention collective de travail. A un moment donné, il crut devoir déclarer la grève et ordonner le piquetage de l'imprimerie. Après quelques semaines, comme elle ne donnait pas de résultats sensibles, cette manoeuvre de grève fut abandonnée; et le syndicat, pour intensifier les effets de l'arrêt de travail, s'est adressé aux fournisseurs et aux clients de la requérante, les informant de la grève et leur demandant soit de ne fournir aucun matériel utilisé pour l'imprimerie, soit de cesser de lui confier leurs travaux d'impression. Les démarches et les représentations des intimés atteignirent leur but. Elles eurent pour résultat que des commandes de la requérante ne furent pas remplies et celle-ci fut ainsi privée de matériaux nécessaires à ses impressions. Plusieurs journaux, dont la liste avait été distribuée aux clients de Verdun Printing and Publishing Inc., commencèrent à leur tour à souffrir du refus de ses clients de fournir des flans, des «mats», des clichés, etc. Quelquesuns ne purent même pas être publiés.

(1) $\mathrm{Ce}$ jugement est reproduit au texte dans Relations Industrielles, juillet 1957, vol. 12, no 3 . pp. 251-253.

\section{LF JUGEMENT}

M. le juge Deslauriers commence par rappeler le droit applicable à l'espèce:

«Les lois de notre pays, particulièrement la Loi des relations ouvrières (S.R.Q., 1941, ch. 162A), reconnaissent le droit de grève qui est, suivant la définition de l'art. 2, par. f de cette loi: «La cessation concertée de travail par un groupe de salariés. »

Cette prise de position est absolument au point; car c'est à loccasion d'une grève, ou comme manoeuvre de grève que le syndicat a boycotté l'imprimerie. Mais notons dès maintenant, quitte à $\mathbf{y}$ revenir plus au long dans nos observations, que le boycottage peut être pratiqué sans que les parties soient en état de grève. Le savant magistrat continuẹ:

«En admettant qu'il y ait, dans le présent cas, une grève légale, l'Union gréviste est-elle demeurée dans les limites permises? 》

Le litige ne pouvait être présenté avec plus de netteté et de clarté: ce qu'il y avait devant le tribunal était un boycottage éminemment dommageable pour la requérante: ce boycottage étaitil illégal? Car un boycottage peut être dommageable et ne pas être illégal, par exemple, s'il reste dans les limites de l'exercice d'un droit. M. le juge Deslauriers écrit alors:

«Le tribunal serait prêt à admettre qu'une certaine espèce du boycottage peut être légale pour promouvoir les intérêts d'un groupe, comme l'en- 
tente parmi certains membres d'une union de cesser de faire affaires avec une personne contre qui une action concertée est dirigée. C'est ce qu'on appelle le primary boycott. Les actes posés par les intimés n'entrent pas dans cette catégorie. Ils s'identifient plutôt avec les actes que l'on qualifie de secondary boycott, soit des machinations faites dans le but de nuire à une personne en forçant d'autres personnes à lui nuire aussi. »

Cette façon de voir est conforme à la plus saine doctrine. Le jugement continue:

«Constatant que la grève à l'atelier n'aurait aucun résultat, l'Union a eu recours à des pratiques dommageables et nuisibles, en intervenant auprès de tierces personnes en relations d'affaires avec la requérante, pour faire cesser ces relations. 》

Vient ensuite la partie du jugement qui porte sur l'injonction. Elle ne présente rien de nouveau. L'injonction a été accordée par appplication de la doctrine bien connue. Le tribunal, dans son pouvoir discrétionnaire, a été d'avis qu'il y aurait beaucoup plus d'inconvénients pour la requérante à ne pas bénéficier d'une injonction interlocutoire que pour l'intimé de voir accorder cette injonction. Sur ce point encore, nous sommes en face d'un bon jugement.

\section{Observations}

Le boycottage a été étudié à Fribourg, aux «Journées suisses de Droit privé 》 tenues par l'Association Henri Capitant ${ }^{2}$, du 6 au 10 juin 1956. Le rapporteur général était $M$. le dayen Henri Deschenaux, de la Faculté de Droit de cette ville de la Suisse francaise, qui écrit dans un questionnaire adressé aux rapporteurs nationaux:

《1. Boycottage: - abstention organisée de relations d'affaires avec un agent économique (industriel, commerçant, employeur, employé) en vue de l'amener, par la limitation ou la paralysie de son activité, à modifier son comportement. Une mise à l'index formelle n'est pas nécessaire; l'isolement du boycotté peut résulter

(2) La publication des travaux des Journées suisses
est actuellement en cours et paraîtra comme est actue
tome $\mathbf{X}$. du seul jeu des contrats d'exclusivité. 》

Ce texte peut servir de définition.

Il y a deux espèces de boycottage: le boycottage dans les conflits entre employeurs et employés ou entre employés dont les uns font pression sur les employeurs pour qu'ils refusent d'employer les autres ou qu'ils les congédient; et le boycottage de la concurrence économique entre industriels ou commerçants.

Le boycottage, qu'il s'agisse du boycottage dans les conflits du travail ou de celui de la concurrence économique, se présente sous différentes formes d'organisation. Il y a le boycottage simple et le boycottage tripartite. Dans le boycottage simple, plusieurs personnes décident d'un commun accord et comme spontanément de s'abstenir de contracter avec une autre. Ainsi, des menuisiers s'unissent pour ne pas travailler chez tel entrepreneur du bâtiment, des marchands détaillants s'engagent à ne pas acheter de tel manufacturier. Certains auteurs disent qu'il n'y a pas à proprement parler de boycottage dans ce que d'autres appellent le boycottage simple.

Dans le boycottage tripartite, le promoteur du boycott, par exemple un syndicat professionnel, obtient d'un groupe de personnes, qu'on appelle les exécutants, disons les membres du syndicat, qu'ils procèdent à l'isolement du boycotté. Nous avons là un cas de véritable boycott.

Le boycottage peut être soit direct, soit indirect. Dans l'exemple que nous venons de donner, où le syndicat professionnel se fait promoteur du boycott avec ses membres comme exécutants et leur employeur comme boycotté, nous avons un boycottage direct. Si les exécutants sont des tiers par rapport au syndicat, par exemple, des fournisseurs du boycotté, nous aurons un boycottage indirect.

Ainsi, dans le boycottage direct, le promoteur et les exécutants, c'est-à-dire les boycotteurs, se trouvent sur un palier économique différent de celui du boycotté. Dans notre exemple, les boycotteurs sont sur le palier du travail et le boycotté est sur le plan de l'industrie ou du commerce. Le boycottage direct est exécuté par ceux auxquels il doit pro- 
fiter. Comme nous l'avons vu, ce boycottage a été déclaré légal par $M$. le juge Deslauriers.

Dans le boycottage indirect, promoteur, bénéficiaires et boycotté sont sur le même palier économique, comme dans le boycottage de concurrence économique entre industriels et commerçants, ou sur des paliers économiques différents, comme dans celui des conflits du travail, mais dans les deux cas, le promoteur du boycottage ou le boycotteur s'adresse à un groupe supérieur ou inférieur pour le lui faire pratiquer; et ainsi, ce boycottage n'est pas exécuté directement par ceux auxquels il doit profiter. L'affaire Verdun était un beau cas de boycottage indirect dans un conflit du travail: le promoteur était le syndicat et les bénéficiaires étaient ses membres, mais ils n'étaient pas les exécutants. Le syndicat s'est adressé aux fournisseurs et aux clients de la requérante pour gu'ils boycottent l'imprimerie au bénéfice de ses membres.

Voici maintenant un cas de boycottage indirect dans la concurrence économique: des commerçants de gros qui veulent agir sur un confrère dissident s'assurent, au besoin par la menace d'un boycottage direct, le concours des fabricants ou des commerçants en détail pour que ceux-ci boycottent le dissident. Ici les promoteurs, qui sont en même temps les bénéficiaires, et le boycotté sont sur le même palier économique, mais les exécutants, les fabricants ou les commerçants de détail, sont les uns d'un échelon supérieur et les autres d'un échelon inférieur au boycotté.

Il y aurait une très belle étude à faire sur l'appréciation juridique du boycottage dans notre droit. Indiquonsen les grandes lignes. D'après la législation et la jurisprudence, est-ce que le boycottage est en principe licite?... Il faut sans hésiter répondre dans l'affirmative. "Le boycottage direct ne semble pas illégal en soi », dit M. le Sénateur Léon Mercier-Gouin ${ }^{3}$. La légalité du boycott ou de la black-list dépend entièrement des actes pratiqués. C'est là une conséquence de la règle de droit qui veut que «the trade-interference

(3) Cours de droit industriel, 2ème éd., tome I, «Lois ourrières 》, p. 196. is not in itself a specific tort 》 ${ }^{4}$. C'est le sens du jugement dans l'affaire Verdun.

Si le boycottage en principe est licite, quand cesse-t-il de l'être?... Il faudrait ici étudier les limites assignées par la loi et la jurisprudence à la constitution et à l'activité des cartels dans l'intérêt de l'économie nationale, en particulier pour lutter contre l'avilissement des prix par le «dumping», aussi dans l'intérêt du public ou de l'ensemble des consommateurs, spécialement la lutte contre le maintien de prix artificiellement élevés. Viendraient ensuite les limites assignées par la loi et la jurisprudence à la constitution et à l'activité des organisations professionnelles, une des principales espèces de boycottage.

Le boycottage indirect a été condammé comme causant un dommage injustifiable à autrui, dit M. Léon MercierGouin ${ }^{5}$. Il ne cite pas de cause. A-t-il à l'idée l'arrêt de la Chambre des Lords dans Quinn vs Leathem ${ }^{6}$ :

«A combination of two or more, without justification or excuse, to injure a man in his trade by inducing his customers or servants to break their contracts with him or not to deal with him or continue in his employment is, if it results in damage to him, actionable. 》

La doctrine que nous trouvons dans cet arrêt est considérée comme applicable ici. Il y a aussi l'affaire Crofter Hand Woven Harris Tweed Co. Ltd vs Veitch ', que l'on invoque souvent en matière de sécurité syndicale et qui a fait l'objet d'un commentaire dans le Canadian Bar Review. 8

Le boycottage, avons-nous dit, peut se pratiquer sans que les intéressés soient en état de grève, donc en dehors des conflits de travail, mais grève et boycottage ont plusieurs règles de droit en commun. La jurisprudence anglaise et américaine leur assigne assez souvent les mêmes limites et la jurisprudence française établit une analogie entre la grève et la mise à l'index ou boycott.

(4) Norman A. Citrine. Trade Union Law. London: Stephens \& Sons Ltd., 1950, p. 55.

(5) loc. cit.

(6) Fc. p. 495.

(7) (1942) A.L.E.R., 142.

(8) 1942 , Vol. 20, p. 636. 
L'étude du boycottage, tel que nous la concevons, porterait enfin sur un examen des conséquences d'un boycottage illicite sur le plan du droit civil, du droit administratif et du droit pénal ou criminel. Il faut espérer qu'un confrère entreprendra un jour cette étude.

A l'occasion d'un arrêt de la Cour de Cassation rapponté sous le titre Commerce et Industrie, liberté, atteinte, entente professionnelle, mise à l'index, faute délictuelle, dommagesintérêts, une note très intéressante a été rédigée pour le Recueil Dalloz par Paul Esmein, professeur honoraire à la Faculté de Droit de Paris. ${ }^{9}$ On y trouve une revue complète de la jurisprudence française. Nous en citons de longs extraits:

«La mise à l'index ou boycottage consiste à refuser de passer des contrats, et à inciter les tiers à refuser d'en passer, avec certaines personnes, à quoi peut s'ajouter la rupture et l'incitation à la rupture de contrats en cours. » nue:

Après cette définition, l'auteur conti-

«La mise à l'index peut avoir lieu dans tous les domaines de rapports entre hommes. Elle a été soumise à l'appréciation des tribunaux surtout dans deux domaines: celui des conflits du travail entre employeurs et employés, ou entre employés dont les uns font pression sur les employeurs pour qu'ils refusent d'embaucher les autres ou pour qu'ils les congédient; et celui de la concurrence économique entre industriels ou commerçants. Or, si l'on examine la jurisprudence dans son ensemble, il apparaît que son attitude est différente, à certains égards, suivant qu'il s'agit des conflits du travail ou des conflits de la concurrence économique.

Depuis que la grève est licite et que les ouvriers sont autorisés à se grouper pour la défense de leurs intérêts professionnels, il n'est plus possible de déclarer illicite la mise à l'index d'un employeur par les ouvriers quand elle a lieu pour atteindre un but qu'il serait licite de cher-

(9) D. 1957, j. 665 cher à atteindre par la grève. La grève est un refus, non de contracter, mais d'exécuter un contrat. La mise à l'index proprement dite, telle qu'elle se présente dans les espèces soumises aux tribunaux, comporte généralement une pression exercée sur des tiers en vue de les amener à rompre leurs relations ou à refuser de contracter avec ceux qui sont mis à l'index. Mais quand, par exemple, un syndicat ouvrier met à l'index les employeurs qui ont refusé d'accepter le tarif des salaires proposé par le syndicat, il y a à la fois ordre aux ouvriers en cours de contrat de faire grève et ordre à tous autres de ne pas s'embaucher chez ces employeurs. On ne peut déclarer illicite le second ondre si le premier est déclaré licite. »

Viennent ensuite plusieurs arrêts sur des conflits du travail, où la mise à l'index a été déclarée tantôt licite et tantôt illicite. Plusieurs portent sur des espèces qui s'apparentent à certaines de nos formules de sécurité syndicale. A la suite de ces arrêts, le professeur Esmein écrit:

«Il est certain cependant que les ouvriers qui ne veulent pas suivre les directives du syndicat peuvent causer préjudice à l'intérêt de la profession, car ceux qui parlent au nom des ouvriers ont d'autant plus de chances de réussir dans leurs revendications qu'ils sont appuyés par un plus grand nombre de gens. Mais la jurisprudence n'a pas suivi ceux qui voulaient permettre aux syndicats de contraindre, par la mise à l'index, les récalcitrants à les suivre, appliquant la doctrine de Rousseau: «Quiconque refusera d'obéir à la volonté générale sera contraint par tout le corps: ce qui ne signifie autre chose sinon qu'on le forcera d'être libre. »

On sait que par la Loi du 2 avril 1956, le législateur français est intervenu pour faire échec aux pressions que les syndicats cherchaient à exercer sur les ouvriers par l'intermédiaire des employeurs.

Cette loi a été insérée au code du travail, liv. III:

« Art. ler a. Il est interdit à tout emploveur de prendre en considération lappartenance à un syndicat on 
l'exercice d'une activité syndicale pour arrêter ses décisions en ce qui concerne notamment l'embauchage, la conduite et la répartition du travail, la formation professionnelle, l'avancement, la rémunération et l'octroi d'avantages sociaux, les mesures de discipline et de congédiement.

«Il est interdit à tout employeur de prélever les cotisations syndicales sur les salaires de son personnel et de les payer au lieu et place de celui-ci.

«Le chef d'entreprise ou ses représentants ne devront employer aucun moyen de pression en faveur ou à l'encontre d'une organisation syndicale quelconque.

«Toute mesure prise par l'employeur contrairement aux dispositions des alinéas précédents sera considérée comme abusive et donnera lieu à dommages-intérêts.

«Ces dispositions sont d'ordre public. 》

Analysant la jurisprudence française sur les conflits du travail, le professeur Esmein écrit:

«Mais il n'apparaît pas que, pour juger si les grèves et les mises à l'index, dans les conflits du travail, sont licites ou illicites, les tribunaux tienment compte de l'intérêt de l'ensemble de la population, de ce que la cour d'appel, dans l'arrêt ci-dessus, appelle l'intérêt public général. L'ensemble de la population a un intérêt majeur à pouvoir se procurer de façon continue tout ce dont elle a besoin, et à un prix en rapport avec ses ressources. Or les grèves et mises à l'index dans les conflits du travail tendent le plus souvent, directement ou indirectement, outre la gêne qu'elles créent pendant leur durée par la privation de produits ou services, à une augmentation de salaires, laquelle à son tour contribue à une augmentation du prix des produits et des services à payer par les consommateurs. Cependant jamais on ne voit cette considération intervenir dans les arrêts pour déclarer illicite une grève ou une mise à l'index dans ce genre de conflits.

Il en est autrement, d'après une série d'arrêts récents, dont l'arrêt cidessus recueilli, qui ont eu à apprécier la licéité d'une mise à l'index dans les conflits de la concurrence économique. »

Le professeur Esmein fait alors une analyse de la jurisprudence de la mise à l'index dans cette deuxième catégorie de conflits. Les arrêts qui y figurent ont peu d'intérêt pour nous parce qu'ils portent en partie sur le code pénal français. Au surplus, cette deuxième catégorie de conflits est étrangère au jugemert que nous étudions.

\section{Certification syndicale et perte de major:té DURANT LES NÉGOCIATIONS}

La perte de la majorité au cours des procédures de négociation peutelle être invoquée pour obtenir de la C.R.O. une annulation d'un certificat de reconnaissance syndicale dûment obtenu? 1

Le 24 janvier 1956, l'Union Ouvrière mise en cause a obtenu sa certification de la Commission de Relations Quvrières de la pravince de Québec, pour représenter les employés de la requérante, sauf les contremaîtres, les vendeurs et

(1) Jugement rendu le 28 juin 1957 par l'honorable juge Ignace-J. Deslauriers, Cour Supérieure, District de Montréal, no $400,271$. Jonergin Co., Inc., requérante, la Commission de relations ouvrières de la province de Québec, intimée, et Montreal Printing Specialties and Paper Products Union, local no 521, miseen-cause. les employés de bureau... Au moment de la certification, suivant les prétentions de la requérante, cette dernière comptait vingt-et-un (21) employés. Depuis ce temps, elle en a embauché deux autres, portant à vingt-trois (23) le nombre de ses employés. Parmi ses employés, elle compte J. Howard, P. Pesant et J. Kusenke. Ces trois personnes sont des artistes, tel qu'elle le déclare sur ses différentes listes d'employés produites et comme il a été admis à lenquête.

L'Union certifiée mise en cause a 